\title{
View Maintenance in Object-Oriented Databases
}

\author{
Reda Alhajj ${ }^{1}$ and Faruk Polat ${ }^{2}$ \\ 1 Department of Computer Engineering and Information Science, Bilkent University, \\ 06533 Ankara, Turkey \\ 2 Department of Computer Engineering, Middle East Technical University, 06531 \\ Ankara, Turkey
}

\begin{abstract}
In this paper, we present a model that facilitates view maintenance within object-oriented databases. For that purpose, we differentiate between two categories of classes, base classes and brother classes. While the former constitute the actual database, the latter are introduced to hold virtual database, i.e., views derived from base classes. To achieve incremental view update, we introduce a modification list into each base class. A series of algorithms are developed to serve the purpose. Finally it happened that, view maintenance within object-oriented databases subsumes that within the nested and hence conventional relational models.
\end{abstract}

Keywords: Algorithms; Base Classes; Modification Lists; ObjectOriented Databases; Views.

\section{Introduction}

Views are derived (virtual) data that can be materialized and subsequently queried against. View maintenance is an important aspect of query models for having a lot of application areas ranging from integrity constraint maintenance to persistent queries among others. However, the difficulty and complexity of a view maintenance approach is dependent on the underlying data model. Although there have been man studies on view maintenance and materialization within the relational model $[7,8]$, much effort is still required on view maintenance within the nested relational model and object-oriented data models in addition to the effort done in this respect e.g. $[1,2,6,9,10,11]$.

In object-oriented databases, through the use of object identifiers and virtual data, the view mechanism can be implemented correctly. Any update made through a view would be an indirect update, because the real data would be accessed through the use of pointers. No replication is necessary, so no inconsistencies due to the generation or updating of views emerge. We argue that deferred update is more reasonable within the object-oriented context, because updates are most of the time due to indirect modifications and hence require much more effort to be reflected into related views. We should not speak about the violation of referential integrity unless objects are accessed. Only at that time references to deleted objects should be recognized and the violation of referential 
integrity should be checked for. Earlier recognition might be time wasting and useless.

In this paper, we describe a model which facilitates view maintenance in object-oriented databases. The basic idea in our approach is to distinguish modifications to objects on which a view is dependent since the last derivation (update) of that view. Therefore, in any subsequent view update only such modifications are considered and hence the number of objects to be accessed while updating a view is reduced. To achieve that, we categorize classes into base classes and brother classes. The latter correspond to view definitions and the former hold the actual database. Further, to each base class we add a modification list which keeps track of all modifications to be reflected on demand to dependent views. Different algorithms have been developed to handle the view maintenance.

The rest of this paper is organized as follows. The basic model on which our work is based, is presented in Section 2. In Section 3, we give the algorithms that govern and guarantee incremental view maintenance and materialization. Section 4 is the conclusions.

\section{The Basic Model}

In this section we introduce the data model that facilitates incremental view maintenance. To start with, any object with an object identifier $o_{i d}$ qualifies to be considered in the set of objects of a class $c$, denoted by $L_{\text {instances }}(c)$, if and only if object $o_{i d}$ understands nothing more than the behavior defined for objects of class $c$. The behavior for class $c$ consists of two parts, inherited behavior and locally defined behavior. So, let $L_{\text {behavior }}(c)$ denotes the local behavior for class $c$. Then, the whole behavior, object instances and attributes for class $c$ are recursively defined based on its direct superclasses, say $\left[c_{p_{1}}, c_{p_{2}}, \ldots, c_{p_{n}}\right]$.

$W_{\text {behavior }}(c)=L_{\text {behavior }}(c)+\sum_{i=1}^{n} W_{\text {behavior }}\left(c_{p_{i}}\right)$

$W_{\text {instances }}(c)=L_{\text {instances }}(c)+\sum_{i=1}^{t} W_{\text {instances }}\left(c_{b_{i}}\right)$

$W_{\text {attributes }}(c)=L_{\text {attributes }}(c)+\sum_{i=1}^{n} W_{\text {attributes }}\left(c_{p_{i}}\right)$

Each method implements a certain function and has a receiver, former parameters and a result. Further, a method is invoked via a corresponding message of the general form $m\left(p_{1}, p_{2}, \ldots, p_{k}\right)$, where $m$ is the method name, $p_{1}$ is the receiver and $p_{2}$ to $p_{k}$ are the parameters.

So, based on what has been defined so far, a class is distinguished by some properties and constructs constituting the class definition. Consequently, consider a general class $\jmath$ to include all such class definitions. Therefore, by definition each object in $L_{\text {instances }}(\jmath)$ holds the definition of at least one class $c$ (this will be clear later in this section). Such object is defined to be a quadrate $\left(C_{p}(c), C_{b}(c), L_{\text {attributes }}(c), L_{\text {behavior }}(c)\right)$. It is obvious that, the definition of class $\jmath$ itself is an object in class $\jmath$.

To surn up, a class $c$ is formally defined as a pair $\left(P_{d}, P_{o}\right)$; where $P_{d}$ is an identifier, it is either the $o_{i d}$ of an object in class $\jmath$ or the identifier of another existing class, say $c^{\prime}$. For the former case, $P_{0}$ refers to a pair $\left(L_{\text {instances }}(c), M_{\text {ist }}(c)\right)$, 
where $M_{l i s t}(c)$ denotes a modification list consisting of modification tuples. For the latter case, on the other hand, $P_{o}$ refers to a view definition; it is a view directly derived from class $c^{\prime}$.

A modification tuple, denoted $M_{\text {tuple }}\left(V_{i d}\right)$, is a quadrate $\left(V_{i d}, O_{\text {inserted }}, O_{\text {deleted }}, O_{\text {updated }}\right)$, where $V_{i d}$ is an identifier of a view derived from class $c ; O_{\text {inserted }}, O_{\text {deleted }}$ and $O_{\text {updated }}$ are respectively the sets of objects inserted into, deleted from and updated in $W_{\text {instances }}(c)$, after the last update of view $V_{i d}$ and before the update of another view based on class $c$. The latter update causes a new modification tuple to be added to the list of class $c$.

A view is a triplet $\left(V_{i d}, W_{\text {instances }}\left(V_{i d}\right), P_{\text {expression }}\left(V_{i d}\right)\right)$, where $W_{\text {instances }}\left(V_{i d}\right)$ is the set of $o_{i d}$ 's for objects from $W_{\text {instances }}\left(c^{\prime}\right)$ matching the conjunction of predicates in $P_{\text {expression }}\left(V_{i d}\right)$. In other words, $P_{\text {expression }}\left(V_{i d}\right)$ is a list of predicates the conjunction of which constitute the predicate expression that filters objects from $W_{\text {instances }}\left(c^{\prime}\right)$ to be included in $W_{\text {instances }}\left(V_{i d}\right)$. Further, $P_{\text {expression }}\left(V_{i d}\right)$ must be updated to reflect schema changes, i.e., if a schema change drops some of the messages incorporated within some predicates in $P_{\text {expression }}\left(V_{i d}\right)$, then those predicates should be adjusted to reflect the change [4]. For a detailed formal definition of predicates see our previous work on query models $[3,5]$.

This way, we differentiate between two categories of classes according to the instantiation of $P_{d} ;$ base classes and brother classes. A base class directly points to a class definition in class $\jmath$, i.e., its $P_{d}$ is the $o_{i d}$ of an object in class $\jmath$. A brother class holds a view definition and indirectly refers to an object in class $\jmath$ via a base class, i.e., its $P_{d}$ is the identifier of the base class from which the view is derived. According to this classification, all brother classes in the database are arranged into sets of equivalent-classes ${ }^{\star \star}$ and hence each group of brother classes sharing the same base class definition constitute an equivalent-class. The cardinality of each equivalent-class is the number of views derived directly from the related base class. On the other hand, the cardinality of the modification list of a class shows the number of views dependent on that class. Further, modification lists hold the update history of the actual database.

To illustrate what has been introduced above, consider the class hierarchy shown in Fig. 2 and the objects shown in Fig. 2. Next to each class in Fig. 2, there are three sets which include $L_{\text {attributes }}, L_{b e h a v i o r}$ and $L_{\text {instances, }}$, respectively. In addition, for every class given in Fig. 2, $C_{p}$ and $C_{b}$ are implicitly indicated via class connections. Some example brother classes are enumerated next.

- Females is a brother class of person with $P_{d}=$ person, $P_{\text {expression }}($ Females $)=\left[\operatorname{sex}(p)=" F^{\prime \prime}\right]^{\dagger}, W_{\text {instances }}($ Females $)=\left\{o_{i d_{2}}, o_{i d_{4}}\right\}$

- BasicCourses is a brother class of course with $P_{d}=$ course,

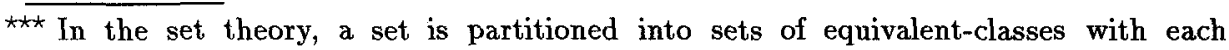
equivalent-class containing related elements; while all the equivalent-classes of a set are pairwise disjoint

$\dagger$ Variable $p$ is used as a pointer to objects in the target base class, here person 
$W_{\text {instances }}(c)$ are performed from within a subclass of class $c$ against objects subsumed in $W_{\text {instances }}(c)$ or else from within another class against shared objects along the class-composition hierarchy. Therefore, locating and controlling global modifications is as important as local modifications and also it is required in preserving database consistency and integrity.

$$
\begin{aligned}
& M_{\text {list }}(\text { person })=[(\text { Females }, \phi, \phi, \phi)] \\
& M_{\text {list }}(\text { staf } f)=\left[\left(\text { Eemales }, \phi, \phi,\left\{o_{i d_{5}}\right\}\right)\right] \\
& \left.M_{\text {list }}(\text { department })=0\right]
\end{aligned}
$$

(a) After the addition of Females view

$\begin{array}{lr}M_{\text {list }}(\text { person })=[(\text { Females }, \phi, \phi, \phi)] & M_{\text {list }}(\text { student })=\left[\left(\text { Females }, \phi,\left\{o_{i d_{4}}\right\}, \phi\right)\right] \\ M_{\text {list }}(\text { staff })=\left[\left(\text { Females }, \phi, \phi,\left\{o_{\text {id }}\right\}\right)\right] & M_{\text {list }}(\text { res-ass })=[(\text { Females }, \phi, \phi, \phi)] \\ M_{\text {list }}(\text { department })=[] & M_{\text {list }}(\text { course })=\left[\left(\text { BasicCourses }, \phi,\left\{o_{i d_{9}}\right\},\left\{o_{i d_{10}}\right\}\right)\right]\end{array}$

(b) After the addition of BasicCourses view

$M_{\text {list }}($ person $)=[($ Females $, \phi, \phi, \phi),($ Staff BrothersOfSusan, $\phi, \phi, \phi)]$

$M_{\text {list }}($ student $)=\left[\left(\right.\right.$ Females $\left.\left., \phi,\left\{o_{i d_{4}}\right\}, \phi\right)\right]$

$M_{\text {list }}($ staff $)=\left[\left(\right.\right.$ Females, $\left.\phi, \phi,\left\{o_{i d_{5}}\right\}\right),($ Staff BrothersOfSusan, $\left.\phi, \phi, \phi)\right]$

$M_{\text {list }}($ res-ass $)=\left[(\right.$ Females $, \phi, \phi, \phi),\left(\right.$ StaffBrothersOf Susan, $\left.\left.\phi, \phi,\left\{o_{i d_{6}}\right\}\right)\right]$

$M_{\text {ist }}($ department $)=[($ Staf $f$ BrothersOf Susan, $\phi, \phi, \phi)]$

$M_{\text {list }}($ course $)=\left[\left(\right.\right.$ BasicCourses, $\left.\left.\phi,\left\{o_{i d_{9}}\right\},\left\{o_{i d_{10}}\right\}\right)\right]$

(c) After the addition of StaffBrothers OfSusan view

Fig. 3. Modification lists of the base classes given in Figure 1

To keep track of global modifications, each addition of a modification tuple to $M_{l i s t}(c)$ triggers the addition of a modification tuple with the same $V_{i d}$ to the list of every class along both subhierarchies rooted at class $c$. Such tuples are utilized to indicate objects to consider from $W_{\text {instances }}(c)$ in the process of updating view $V_{i d}$. To illustrate this, shown in Fig. 3 are the modification lists of the base classes given in Fig. 2, after the addition of the example brother classes introduced in Section 2. First, we assume that after defining the Females view, object $o_{i d_{4}}$ is deleted from the student class and object $o_{i d_{5}}$ is updated in the staff class; this is reflected into the modification lists shown in Fig. 3(a). Second, as shown in Fig. $33(\mathrm{~b})$, after the BasicCourses view is generated, object $o_{i d_{9}}$ is deleted from the course class and object $o_{i d_{10}}$ is updated in the course class. Finally, in Fig. 3(c); it is shown that after the StaffBrothersOfSusan view is generated, object $o_{i d_{6}}$ is updated in the research-assistant class.

Modifications along the inheritance hierarchy are located by recursively tracing the $C_{b}$ 's of classes along the inheritance subhierarchy rooted at class $c$. On the other hand, to successfully reflect modifications along the class-composition subhierarchy, it is necessary to locate modified objects in each particular class along that subhierarchy and to backtrack (mostly by utilizing an index) to locate in $W_{\text {instances }}(c)$ objects referencing such modified objects. We accomplish this by introducing two general base classes into the class hierarchy, namely NEsting 
of Classes (NEC) and Complex Objects References (COR), to keep track of the relationships between classes and objects, respectively.

Explicitly, $N E C$ holds all class-to-class relationships along the class composition hierarchy, i.e., a relationship between two classes $c_{i}$ and $c_{j}$ is included in $N E C$ to show that class $c_{i}$ has an attribute the value of which is drawn from $W_{\text {instances }}\left(c_{j}\right)$. When a relationship between two classes $c_{i}$ and $c_{j}$ is registered in $N E C$, the relationships between their corresponding objects is reflected into $C O R$ to show that object $o_{i d_{j}}$ from class $c_{j}$ is contained in the state of object $o_{i d_{i}}$ from class $c_{i}$. The definitions in class $\jmath$ for $N E C$ and $C O R$ classes are given in Fig. 3. On the other hand, shown in Fig. 3 are the objects contained in $N E C$ and $C O R$ classes, as the example classes given in Fig. 2 and the corresponding objects shown in Fig. 2 are concerned. This is achieved because in our query model $[3,5]$ we allow the specification of the result of a recursive query to be a subset of the transitive closure.

- $C_{p}(N E C)=\phi, \quad \quad-C_{b}(N E C)=\phi$,

- Lattributes $(N E C)=\{\text { LeftClass:C, RightClass:C }\}^{\ddagger}$

- Lbehavior $(N E C)=\{$ FindClassCompositionHierarchy() $\}$

- $C_{p}(C O R)=\phi, \quad$ - $C_{b}(C O R)=\phi$,

- Lattributes $(C O R)=\left\{\text { LeftObject: } O_{I D}, \text { RightObject: } O_{I D}\right\}^{\S}$

- $L_{b e h a v i o r}(C O R)=\{$ FindReferencingObjects $($ TargetClass $)\}$

Fig. 4. Definitions of classes $N E C$ and $C O R$ in class $J$

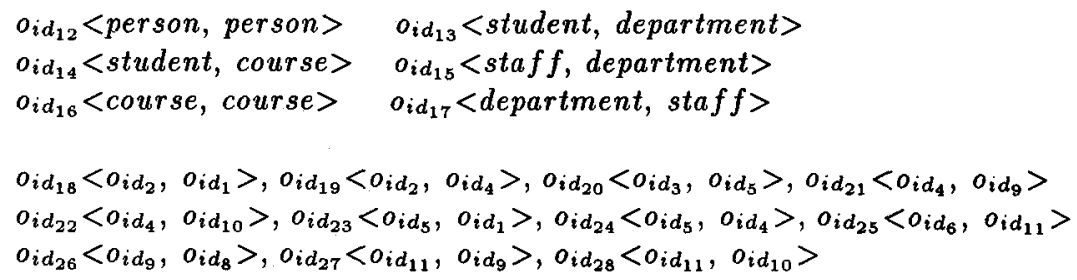

Fig. 5. Objects in $N E C$ and $C O R$ classes

Objects in the two classes NEC and COR are utilized by the algorithms given next in this section. According to Algorithm 3.2, related to a given view $V_{i d}$ which is a brother class of base class $c$, modification tuples in each class along the class-composition subhierarchy rooted at class $c$ are located by tracing the hierarchy in the forward direction starting with class $c$ and using objects in $N E C$. After that, COR is utilized to trace in the backward direction every object in the sets included within the located tuples to determine referencing objects 
in $W_{\text {instances }}(c)$. So, given view $V_{i d}$, Algorithm 3.2 determines three sets of $o_{i d}$ 's for objects to be utilized in Algorithm 3.3 which updates that view. These sets $W_{O_{\text {inserted }}}, W_{O_{\text {deleted }}}$ and $W_{O_{\text {updated }}}$ contain respectively objects which are locally or globally inserted into, deleted from and updated within $W_{\text {instances }}(c)$ since the last update of view $V_{i d}$. To have every modified object located, Algorithm 3.1 is utilized to determine modifications along the inheritance subhierarchy rooted at a particular class.

Algorithm 3.1 (IMT)

$1^{*}$ This algorithm considers only classes along the inheritance subhierarchy rooted at class $c$. The target is to determine modifications to objects in $W_{\text {instances }}(c)$, i.e., objects inserted into, deleted from or updated within $W_{\text {instances }}(c)$ since the last update to view $V_{\text {id }}$ which directly or indirectly depends on base class $c$.

Input: $a$ class $c$ and a view $V_{i d}$.

Output: sets of oid's $C_{O_{\text {inserted }}}, C_{O_{\text {deleted }}}, C_{o_{\text {updated }}}$ of modified objects in $W_{\text {instances }}(c)$.

Steps:

Let $C_{O_{\text {inserted }}}=C_{O_{\text {deleted }}}=C_{O_{\text {updated }}}=\phi$

Let $C_{\text {inheritance }}=[c]$

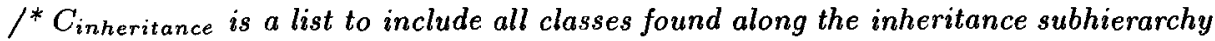
rooted at class $c$.

Let $i=0$

While not end of $C_{\text {inheritance }}$ do

Let $c^{\prime}=C_{\text {inheritance }}[i]$

$C_{\text {inheritance }}=C_{\text {inheritance }}+C_{b}\left(c^{\prime}\right)$

Find $M_{\text {tuple }}\left(V_{i d}\right)$ within $M_{\text {list }}\left(c^{\prime}\right)$.

While not end of $M_{\text {list }}\left(c^{\prime}\right)$ do

. $C_{O_{\text {inserted }}}=C_{O_{\text {inserted }}} \bigcup O_{\text {inserted }}$

- $C_{O_{\text {deleted }}}=C_{O_{\text {deleted }}} \cup O_{\text {deleted }}$

End While

- $C_{O_{\text {updated }}}=C_{O_{\text {updated }}} \cup O_{\text {updated }}$

If there exists an immediate predecessor of $M_{\text {tuple }}\left(V_{i d}\right)$ in $M_{\text {list }}\left(c^{\prime}\right)$ then

- Merge $M_{\text {tuple }}\left(V_{i d}\right)$ with its immediate predecessor

Else

EndIf

. Delete $M_{\text {tuple }}\left(V_{i d}\right)$

Append $\left(V_{i d}, \phi \phi \phi\right)$ at the rear of $M_{l i s t}\left(c^{\prime}\right)$

$i=i+1$

End While

EndAlgorithm

Algorithm $3.2(I D U)$

$/^{*}$ To determine since the last update to view $V_{i d}$, the sets of objects inserted into, deleted from or updated within $W_{\text {instances }}(c)$, where $c$ is the base class $c$ of which view $V_{i d}$ is a brother class.

Input: $V_{\text {id }}$ of a view which is a brother class of base class $c$.

Output: Sets of objects $W_{O_{\text {inserted }}}, W_{O_{\text {deleted }}}$, 
$W_{O_{\text {updated }}}$ to be utilized in Algorithm 3.3 for incremental update of view $V_{i d}$

\section{Steps:}

Let $W_{O_{\text {inserted }}}=W_{O_{\text {deleted }}}=W_{O_{\text {updated }}}=\phi$

Find $M_{\text {tuple }}\left(V_{i d}\right)$ within $M_{\text {list }}(c)$.

If $M_{\text {tuple }}\left(V_{i d}\right)$ is not found then

$\digamma^{*}$ derive view $V_{i d}$ from scratch; it is a new view

Else

$$
\begin{aligned}
& W_{O_{\text {inserted }}}=W_{\text {instances }}(c) \\
& W_{O_{\text {deleted }}}=W_{O_{\text {updated }}}=\phi
\end{aligned}
$$

$$
\begin{aligned}
& \text { Perform } I M T\left(V_{i d}, c\right) \\
& W_{O_{\text {inserted }}}=C_{O_{\text {inserted }}} \\
& W_{O_{\text {deleted }}}=C_{O_{\text {deleted }}} \\
& W_{O_{\text {updated }}}=C_{O_{\text {updated }}} \\
& C_{c c h}=\text { FindClassCompositionHierarch }(c) /{ }^{*} C_{c c h} \text { is a list to include classes }
\end{aligned}
$$
within the class-composition subhierarchy rooted at class $c$.

$$
\text { Let } G=\phi
$$

$/^{*} G$ is a set to include all modified objects along the class-composition subhierarchy rooted at class $c$.

$$
\begin{aligned}
& \text { For every class } c_{i} \text { in } C_{c c h} \text { do } \\
& \text { - Perform IMT( } \left.V_{i d}, c_{i}\right) \\
& \text {. } G=G \bigcup C_{O_{\text {inserted }}} \bigcup C_{O_{\text {deleted }}} \cup C_{O_{\text {updated }}} \text { EndFor } \\
& \text { For every object } o_{i d} \text { in } G \text { do } \\
& \text {. } W_{O_{\text {updated }}}=W_{O_{\text {updated }}}+ \\
& \text { FindReferencingObjects }\left(o_{i d}, c\right)
\end{aligned}
$$

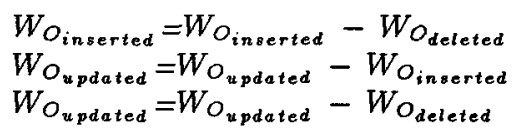

EndIf

\section{EndAlgorithm}

\section{Algorithm 3.3 (ViewUpdate)}

$/^{*}$ To utilize modified objects within $W_{\text {instances }}(c)$ in deriving (updating) view $V_{\text {id }}$ which is a brother class of base class $c$.

Input: $V_{i d}$ of a view which is a brother class of

$$
\text { base class } c \text {. }
$$

Output: $W_{\text {instances }}\left(V_{i d}\right)$

\section{Steps:}

$$
\begin{aligned}
& \text { Perform } I D U\left(V_{i d}\right) \\
& W_{\text {instances }}\left(V_{i d}\right)=W_{\text {instances }}\left(V_{i d}\right)-W_{O_{\text {deleted }}} \\
& W_{\text {instances }}\left(V_{i d}\right)=W_{\text {instances }}\left(V_{i d}\right)-W_{O_{\text {pdated }}} \\
& W_{O_{\text {inserted }}}=W_{O_{\text {inserted }}} \bigcup W_{O_{\text {updated }}} \\
& \text { For every object o in } W_{O_{\text {inserted }}} \text { do } \\
& \quad \text { If oid satisfies } P_{\text {expression }}\left(V_{i d}\right) \text { then } \\
& \quad W_{\text {instances }}\left(V_{i d}\right)=W_{\text {instances }}\left(V_{i d}\right) \bigcup\left\{o_{i d}\right\} \\
& \quad \text { EndIf }
\end{aligned}
$$

\section{Endfor}

EndAlgorithm 
$M_{\text {list }}($ person $)=[($ StaffBrothersOfSusan, $\phi, \phi, \phi), \quad($ Females, $\phi, \phi, \phi)]$

$M_{\text {list }}($ student $)=[($ Females $, \phi, \phi, \phi)]$

$M_{\text {list }}($ staff $)=[($ StaffBrothersOfSusan, $\phi, \phi, \phi),($ Females, $\phi, \phi, \phi)]$

$M_{\text {list }}($ res-ass $)=\left[\left(\right.\right.$ StaffBrothersOfSusan $\left.\phi, \phi,\left\{o_{i d_{6}}\right\}\right),($ Females, $\left.\phi, \phi, \phi)\right]$

$M_{\text {list }}($ department $)=[($ StaffBrothersOfSusan, $\phi, \phi, \phi)]$

$M_{\text {list }}($ course $)=\left[\left(\right.\right.$ BasicCourses, $\left.\left.\phi,\left\{o_{i d_{9}}\right\},\left\{o_{i d_{10}}\right\}\right)\right]$

Fig. 6. Modification lists of the base classes given in Figure 1 after the update of Females view

$M_{\text {list }}($ person $)=[($ Females $, \phi, \phi, \phi),($ StaffBrothers $O f S u s a n, \phi, \phi, \phi)]$

$M_{\text {list }}($ student $)=\left[\left(\right.\right.$ Females $\left.\left., \phi,\left\{o_{i d_{4}}\right\}, \phi\right)\right]$

$M_{\text {list }}($ staff $)=\left[\left(\right.\right.$ Females, $\left.\phi, \phi,\left\{o_{i d_{5}}\right\}\right),($ StaffBrothersOfSusan, $\left.\phi, \phi, \phi)\right]$

$M_{\text {list }}($ res-ass $)=\left[\left(\right.\right.$ Females, $\left.\phi, \phi,\left\{o_{i d_{6}}\right\}\right),($ StaffBrothers $O$ fSusan, $\left.\phi, \phi, \phi)\right]$

$M_{\text {list }}($ department $)=[($ StaffBrothersOfSusan, $\phi, \phi, \phi)]$

$M_{\text {list }}($ course $)=\left[\left(\right.\right.$ BasicCourses $\left.\left., \phi,\left\{o_{i d_{9}}\right\},\left\{o_{i d_{10}}\right\}\right)\right]$

(a) Before the update of Females view

$M_{\text {list }}($ person $)=[($ Females $, \phi, \phi, \phi),($ StaffBrothersOfSusan, $\phi, \phi, \phi)]$

$M_{\text {list }}($ student $)=[($ Females $, \phi, \phi, \phi)]$

$M_{\text {list }}($ staff $)=[($ Females, $\phi, \phi, \phi),($ Staff Brothers OfSusan, $\phi, \phi, \phi)]$

$M_{\text {list }}($ res-ass $)=[($ Females, $\phi, \phi, \phi),($ StaffBrothersOfSusan, $\phi, \phi, \phi)]$

$M_{\text {list }}($ department $)=[($ StaffBrothersOfSusan, $\phi, \phi, \phi)]$

$M_{\text {list }}($ course $)=\left[\left(\right.\right.$ BasicCourses $\left.\left., \phi,\left\{o_{i d_{9}}\right\},\left\{o_{i d_{10}}\right\}\right)\right]$

(b) After the update of Females view

Fig. 7. Modification lists of the base classes given in Figure 1 after the update of StaffBrothers OfSusan view

To illustrate the already introduced algorithms, assume that it is desired to access the Females view. However, accessing a view results in updating its objects because we employ deferred update. Consequently, Algorithm 3.3 is executed and hence Algorithms 3.2 and 3.1, since Algorithm 3.3 calls Algorithm 3.2 which, in its turn, calls Algorithm 3.1. So, on executing ViewUpdate(Females), the related modification lists shown in Fig. 3(c) are traced by Algorithms 3.2 and 3.1 to locate modified objects in $W_{\text {instances }}$ (person); because person is the base class of which Females is a brother class. The following modification sets are returned by Algorithms 3.2:

$W_{o_{\text {inserted }}}=\phi, W_{\text {odeleted }}=\left\{o_{i d_{4}}\right\}, W_{o_{\text {updated }}}=\left\{o_{i d_{5}}, o_{i d_{6}}\right\}$. Algorithms 3.3 utilizes these modification sets to return $W_{\text {instances }}($ Females $)=\left\{o_{i d_{2}}\right\}$. To mark the starting point of the forthcoming update of the Females view, Algorithm 3.1 updates the utilized modification lists from Fig. 3(c) into the lists shown in Fig. 3. To realize the change in the lists more explicitly, consider the execution of ViewUpdate(StaffBrothersOfSusan) by utilizing the lists in Fig. 3(c) and Fig. 3, i.e., without executing ViewUpdate(Females) and after executing ViewUpdate(Females), respectively. Modification lists of the base classes after 
such executions are shown in Fig. 3(a) and (b), respectively. Notice how in Fig. $3(\mathrm{a}), o_{i d_{6}}$ moved within $M_{\text {list }}$ (research-assistant).

\section{Conclusions}

In this paper, we presented a model that facilitates incremental view maintenance within object-oriented databases. Instant reflection of class updates into dependent views is not only time consuming, but also proved to be useless and hence time wasting. View maintenance within the object-oriented context is more challenging than that within the relational model. Thus, our algorithms serve more than the requirements of the relational model. Explicitly speaking, Algorithms 3.1, 3.2 and 3.3 are applicable for both the nested relational model and the relational model as well.

\section{References}

1. Abiteboul, S., Bonner, A.: Objects and Views. Proceedings of the ACM-SIGMOD International Conference on Management of Data (1991)

2. Alashqur, A., Su, S.Y., Lam, H.: OQL: A Query Language for Manipulating Object-Oriented Databases. Proceedings of the $15^{\text {th }}$ International Conference on Very Large Databases. Amsterdam (August 1989)

3. Alhajj, R., Arkun, M.E.: A Query Model for Object-Oriented Database Systems. Proceedings of the $9^{\text {th }}$ IEEE International Conference on Data Engineering. Vienna (April 1993)

4. Alhajj, R., Polat, F.: An Object-Oriented Query Model Enforcing Closure and Reusability. Journal of Mahtematical Modeling and Computing 6 (April 1996)

5. Alhajj, R., Polat, F.: Closure Maintenance in an Object-Oriented Query Model. Proceedings of the ACM International Conference on Information and Knowledge Management. Maryland (November 1994)

6. Dayal, U.: Queries and Views in an Object-Oriented Data Model. Proceedings of the $2^{\text {nd }}$ International Workshop on Database Programming Languages (June 1989)

7. Gupta, A., Mumick, I., Subrahmanian, V.: Maintaining Views Incrementally. Proceedings of the ACM-SIGMOD International Conference on Management of Data. Washington D.C. (1993)

8. Hanson, E.N.: A Performance Analysis of View Materialization Strategies. Proceedings of the ACM-SIGMOD International Conference on Management of Data (1987)

9. Heiler, S., Zdonik, S.B.: Object Views: Extending the vision. Proceedings of the $6^{\text {th }}$ IEEE International Conference on Data Engineering. Los Algeles (February 1990)

10. Kifer, M., Kim, W., Sagiv, Y.: Querying Object-Oriented Databases. Proceedings of ACM-SIGMOD International Conference on Management of Data. San Diego CA (June 1992)

11. Rundensteiner, E.A.: A Methodology for Supporting Multiple Views in ObjectOriented Databases. Proceedings of the $18^{\text {th }}$ International Conference on Very Large Databases. Vancouver BC (August 1992) 